\title{
In-screw polymethylmethacrylate- augmented sacroiliac screw for the treatment of fragility fractures of the pelvis: a prospective, observational study with 1-year follow-up
}

\author{
Andreas Höch $^{1 *}$ (D), Philipp Pieroh ${ }^{1,2}$, Ralf Henkelmann ${ }^{1}$, Christoph Josten ${ }^{1}$ and Jörg Böhme ${ }^{1,3}$
}

\begin{abstract}
Background: The incidence of pelvic ring fractures in the elderly significantly increased. Because of persistent pain and immobilization associated with this injury, surgical treatment is recommended. To minimise comorbidities and surgical risk, percutaneous techniques are becoming more relevant. In-screw cement augmentation of sacroiliac screw fixation is a promising procedure; however, clinical follow-up data remain scarce. This study investigated the safety and possible complications of the procedure along with a 1-year follow-up.

Methods: Thirty-four patients (treated with 43 screws) were prospectively included. Data on patients' age and sex, the mechanism of accident, fracture pattern, duration of hospital stay, surgery and adverse events were recorded. Data were obtained postoperatively on the reduction of pain and complications, such as infection, cement leakage and neurological deficits, and at 1-year follow-up on pain, quality of life according to the 12-Item Short Form Survey and mobility. Implant failure was defined as retraction or dislocation of screws and was also documented.

Results: Screw-related complications occurred with 2 of 43 screws. None of these complications were related to cement augmentation. In-hospital adverse events occurred in 6 of 34 patients. Postoperative pain, measured by the visual analogue scale, was significantly reduced from $6.7 \pm 1.4$ preoperatively to $2.7 \pm 1.0$ postoperatively $(p<0.001)$. Although patients complained of pain at the 1-year follow-up, they reported a significant decline compared with pain at admission $(3.4 \pm 2.3 ; p<0.001)$. Results on the quality of life were comparable with those for the age- and gender matched German population. All patients were mobile, and no implant failure was detected.

Conclusions: The results indicate that in-screw augmented sacroiliac screw fixation for fragility fractures of the pelvis is a safe technique. Pain was significantly reduced immediately after surgery compared to the preoperative state. Furthermore, significant pain reduction after one year compared to the preoperative state and quality of life was comparable to the age- and gender- matched German population. Thus, we recommend in-screw augmentation for screw fixation for sacral fragility fractures of the pelvis following failed conservative treatment.
\end{abstract}

Keywords: Fragility fracture, Pelvic fracture, Sacrum fracture, Polymethylmethacrylate, Sacroiliac screw fixation

\footnotetext{
* Correspondence: andreas.hoech@medizin.uni-leipzig.de

${ }^{1}$ Department of Orthopedics, Trauma and Plastic Surgery, Spine Center,

University Hospital of Leipzig, Liebigstraße 20, 04103 Leipzig, Germany

Full list of author information is available at the end of the article
}

(c) The Author(s). 2017 Open Access This article is distributed under the terms of the Creative Commons Attribution 4.0 International License (http://creativecommons.org/licenses/by/4.0/, which permits unrestricted use, distribution, and reproduction in any medium, provided you give appropriate credit to the original author(s) and the source, provide a link to the Creative Commons license, and indicate if changes were made. The Creative Commons Public Domain Dedication waiver (http://creativecommons.org/publicdomain/zero/1.0/) applies to the data made available in this article, unless otherwise stated. 


\section{Background}

The incidence of pelvic ring fractures in elderly patients has been increasing because of the ageing of the population [1-4]. In general, these fractures are associated with the combination of a low-energy trauma and an impaired bone stock $[5,6]$. The predominant fracture morphology is a lateral compression fracture that involves the anterior pubic rami and an impression of the sacral massa lateralis [7]. Based on the increase of these fractures, Rommens and Hofmann established a new classification system to morphologically describe fragility fractures of the pelvis [8, 9]. Apart from causing pain and related immobilisation, lateral compression injuries have a tendency of becoming unstable during conservative treatment $[3,5,10]$. In addition to appropriate osteoporosis therapy and the use of painkillers, several authors recommend surgical treatment $[2,9,11,12]$.

Because of the high rate of comorbidities, postoperative infections and poor bone quality, percutaneous techniques combined with cement application have become more relevant [13-16]. In-screw cement augmentation with fenestrated screws yielded promising results in short-term follow-up examinations [14, 17]. However, long-term follow-up data remain lacking. The present study investigated pain, quality of life, mobility and implant failure in elderly patients who underwent in-screw polymethylmethacrylate (PMMA) augmented sacroiliac (SI) screw fixation at 1-year follow-up.

\section{Methods}

\section{Ethical approval}

The study was approved by the local ethics committee (297-12-24,092,012), and informed signed consent was obtained from all participating patients.

Patients, study design and preoperative data acquisition

The present study was carried out from July 2012 to December 2014. The study protocol is presented in Fig. 1. All patients suffering from a transalar fragility fracture of the sacrum classified as AO 61-B2.1, AO 61-B3.3, FFP IIb FFP IIc, FFP IVb or Denis I bilateral (Table 1) [7, 8] were evaluated. Initially all these patients were treated conservatively with physical therapy allowing full weight bearing. Painkillers were given according to the World Health Organization (WHO) analgesic ladder step 2. If mobilization was adequate, conservative treatment was continued (Fig.1). In cases of persistent immobilization surgical treatment was indicated. Patients from this patient population planned for inscrew PMMA augmented SI screw fixation independent of anterior fracture fixation, without additional posterior fixation (e.g. spinopelvic fixation) were asked for study participation and included in the study.

Thus, 34 patients $(79 \pm 8.25$ years, 32 female and 2 male) were prospectively included. Nine patients sustained a bilateral Denis I fracture. Furthermore, in cases of unilateral sacral fractures, the corresponding anterior fracture was ipsilateral in 11 patients and contralateral in 10 patients.

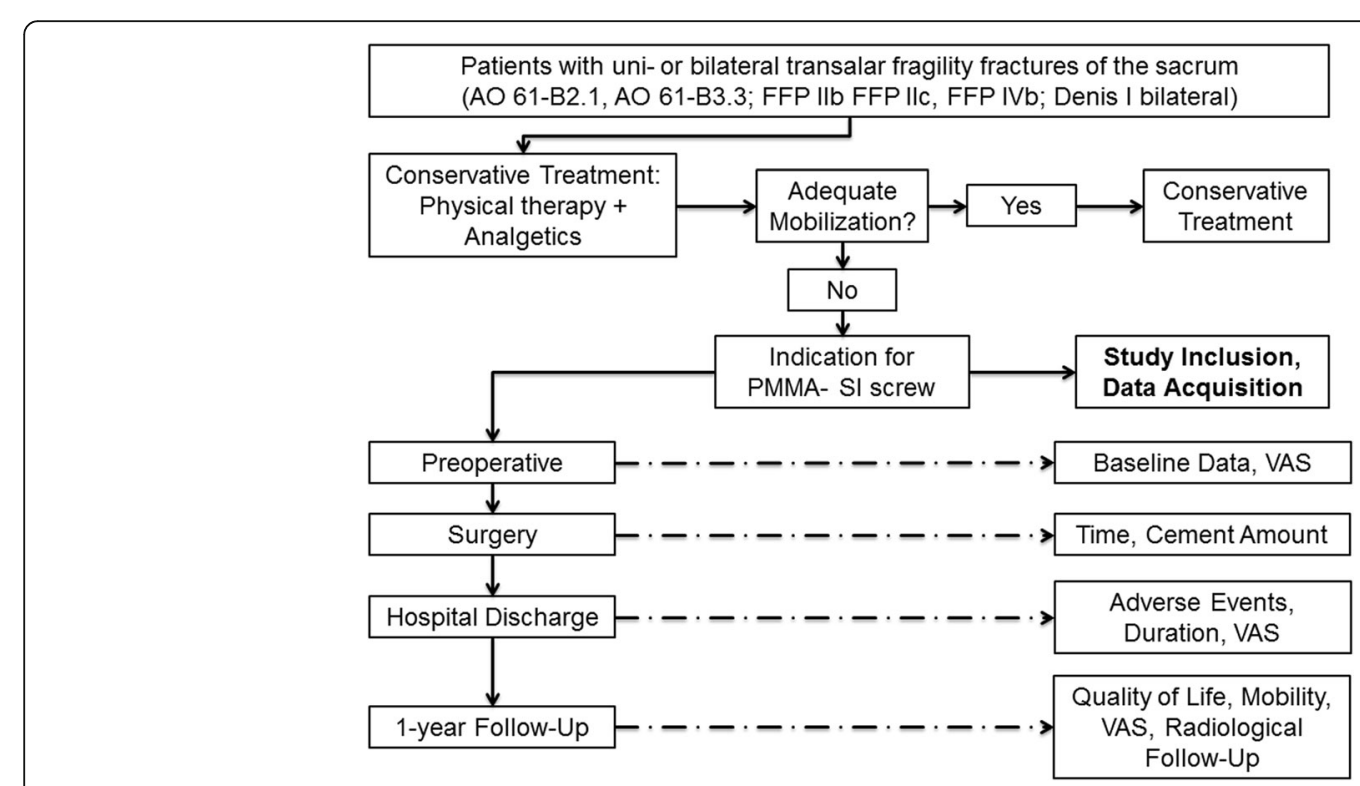

Fig. 1 Study protocol, patient selection and data collection. All patients suffering from a uni- or bilateral transalar fagility fracture of the sacrum were treated primarily conservatively with physical therapy (full weight bearing) and painkillers in accordance to the World Health Organization (WHO) analgesic ladder step 2. If adequate mobilization was achieved, conservative treatment was continued. In cases of persistent immobilization the surgical treatment was indicated. Patients treated posteriorly solely by a PMMA augmented SI screw without additional posterior stabilization and independent of the anterior stabilization were asked preoperatively to participate in the present study. Data were collected at the indicated time points prospectively and reviewed retrospectively 
Table 1 Classification of included pelvic ring fractures according to OTA/AO and Rommens and Hofmann's classification of fragility fractures of the pelvis (FFP) [8]

\begin{tabular}{lll}
\hline Classification & Fracture type & Number of patients \\
\hline OTA/AO & B 2.1 & 25 \\
& B 3.3 & 8 \\
Isolated sacral fracture & Denis I bilateral & 1 \\
FFP & FFP 2b & 11 \\
& FFP 2c & 14 \\
& FFP 4b & 8 \\
& No classification possible & 1 \\
\hline
\end{tabular}

Four patients had a bilateral anterior fracture. Fracture classifications according to the Orthopaedic Trauma Association/Association for the Study of Internal Fixation (OTA/AO) and Rommens et al. are summarized in Table 1. Baseline data such as age, sex and type of admission, the mechanism of accident, injury pattern and comorbidities are presented in Table 2. Thirty-one patients had a history of minor trauma or no trauma. Eleven patients suffered from additional injuries (5 upper limb fractures, 3 osteoporotic spine fractures and 3 cerebral contusions). Moreover, preoperatively the pain level using the visual analogue scale (VAS) was recorded.

\section{Surgery- surgical technique, surgical time and amount of used bone cement}

With the patient under anaesthesia, the insertion point was determined in lateral sacral fluoroscopic views. All

Table 2 Demographic data on age, sex, type of admission, comorbidities, mechanism of accident and injury pattern of the study population

\begin{tabular}{ll}
\hline & Number of patients \\
\hline Age (years) & 34 \\
Gender & $79 \pm 8.25$ (41-92) \\
& 32 females, 2 males; 16:1 \\
Admission & 13 primary admission \\
& 21 secondary transfer \\
& 1 with no comorbidities \\
Comorbidities $^{\text {a }}$ & 24 with $\leq 3$ comorbidities \\
& 9 with $>3$ comorbidities \\
& 28 fell from a stand \\
Mechanism of accident & 3 with unknown/no trauma \\
& 1 because of a traffic accident \\
& 2 fell from a height of $>3$ m \\
& 21 with isolated pelvic ring fracture \\
& 10 with accompanying injuries (ISS < 16) \\
& 3 with multiple injured (ISS > 16)
\end{tabular}

ahypertension, diabetes mellitus, cold, cardiac event in history, neurological event with residual screws were introduced in S1, and the correct course of the threaded K-wire was controlled with fluoroscopic inlet and outlet views [18]. The endpoint was a few millimetres over the sacral midline (Fig. 2a-b).

The screw length was measured using a length gauge over the K-wire. The K-wire was used as a guide, and a cannulated fully threaded 7.5-mm screw (Königsee Implants, Allendorf, Germany) with a washer was introduced. Afterwards, the K-wire was removed, and the bone filler system (Königsee Implants) was applied to inject PMMA (Medtronic, Santa Rosa, CA, USA) under fluoroscopic control. Generally, $3 \mathrm{ml}$ of PMMA was used and spread out on the top and the perforations of the screw (Fig. 2c-e). Data of the surgical procedure including time for surgery, time to surgery, number of screws, additional anterior fixation and the used amount of cement were recorded for further analyses.

\section{Postoperative in-hospital stay - Postoperative pain, duration} of hospital stay, adverse events

After surgery, X-rays (anterior-posterior [AP], inlet and outlet) were taken to verify the position of the implant and exclude cement leakage. CTs were indicated if postoperative neurological deficit, suspected cement leakage or thromboembolism was present.

Postoperatively patients were allowed for full weight bearing with the assistance of a physiotherapist. Supporting antiosteoporotic therapy was given or optimized for all patients according to german national guidelines [19]. Postoperative pain, duration of hospital stay, adverse events and pain using the VAS were determined.

\section{One-year follow-up}

At the 1-year follow-up (range, 12-14 months), intensity of pain according to the VAS, mobility regarding the usage of orthopaedic aids and quality of life using the 12-Item Short Form Survey (SF-12) were investigated. Radiological examinations were performed in AP, inlet and outlet X-rays to analyse implant positioning and implant failure, which was defined as screw retraction or dislocation.

\section{Data analysis}

All data were collected prospectively and retrospectively analysed. Data are presented as mean \pm standard deviation or as medians and ranges according to the distribution type. Statistical analyses were performed using the SPSS 20.0 for Windows (SPSS, Chicago, IL, USA); Student $t$-test and Wilcoxon test were used for analysing data with Gaussian distribution and those with nonGaussian distribution, respectively. $P$ values of $\leq 0.05$ were considered to be statistically significant. 


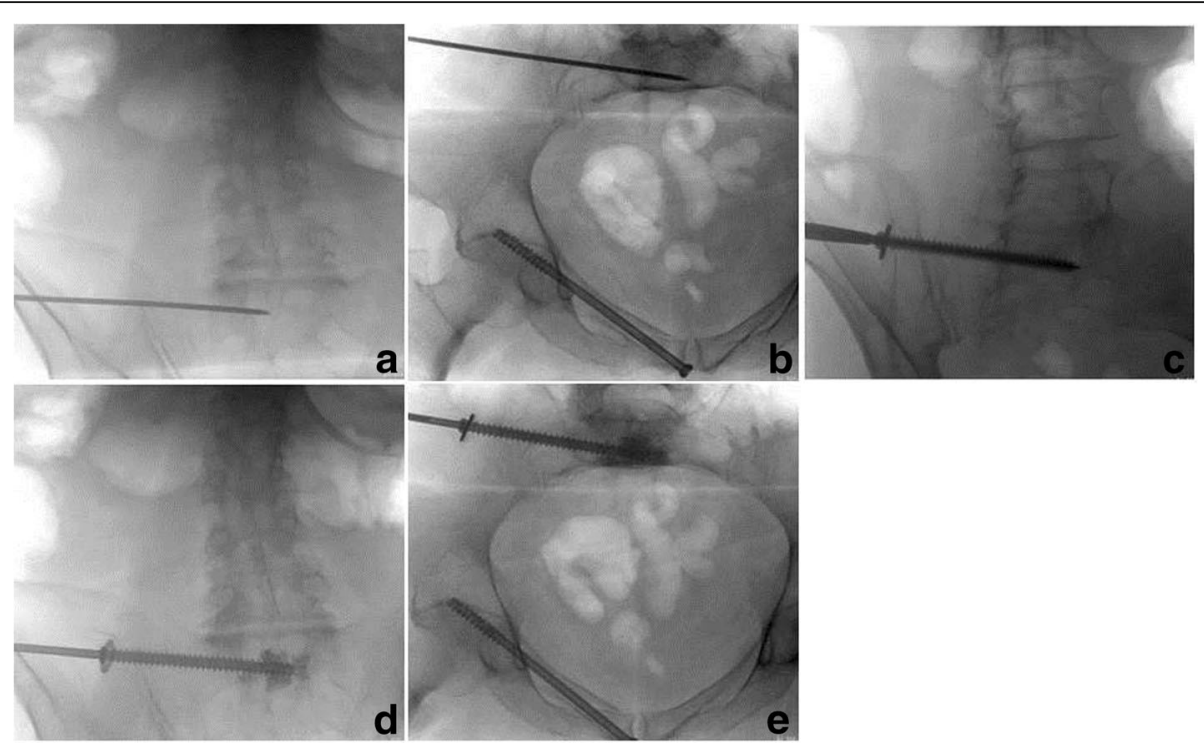

Fig. 2 Surgical technique of in-screw cement-augmented SI screw fixation into S1 with the placement of the K-wire on the right position in inlet and outlet controls (a and b). Screw placement (c) and augmentation with bone-filler device in inlet and outlet views (d and $\mathbf{e}$ )

\section{Results}

\section{Surgical treatment}

The mean time to surgery was $4.5 \pm 2.8$ days after admission. In 25 cases, unilateral SI screw fixation with in-screw cement augmentation was performed in S1. In one case, fixation of two unilateral screws in S1 was performed, and in eight cases, bilateral SI screw fixation (for a total of 43 SI screws) was performed. The mean surgical time was $27 \mathrm{~min}$ per screw, with a range from 17 to 46 min per screw. A mean of $3.0 \pm 0.6 \mathrm{ml}$ of bone cement was used for augmentation (Fig. 3a-d).

Anterior stabilisation was performed either by open reduction and plate osteosynthesis (16 patients) or in cases of low displacement, by retrograde percutaneous screw fixation (16 patients). One patient received a retrograde screw on one side and plate osteosynthesis on the other side.

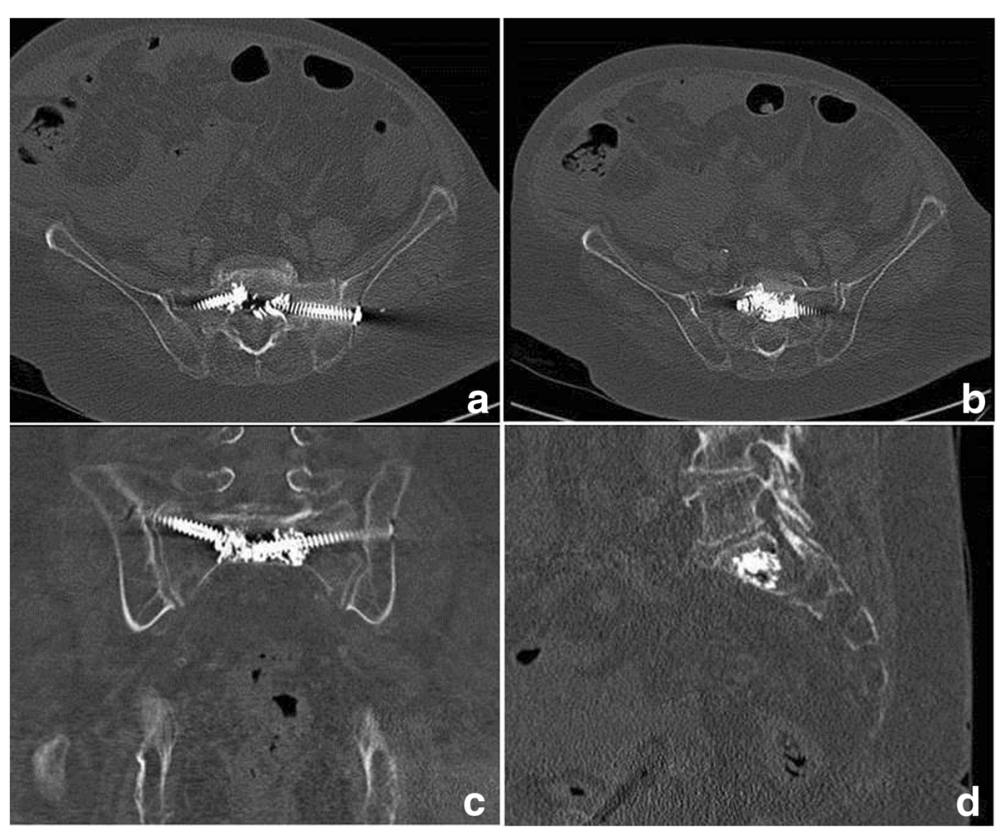

Fig. 3 Postoperative pelvic CT of in-screw cement-augmented SI screw fixation in axial (a and b), coronal (c) and sagittal (d) views 
In-hospital stay- hospital stay and adverse events

The mean duration of hospital stay was $14.1 \pm 6.1$ days (9.6 \pm 6.3 days after surgical treatment). Three patients required intensive care monitoring postoperatively. In two patients, revision surgery was necessary because of the PMMA-augmented SI screw fixation. In one patient, one screw was malpositioned and perforated the ventral alar cortical border close to the iliac vessels, as shown by the inlet X-ray views and subsequent $\mathrm{CT}$. In another patient, postoperative hematoma in the gluteal muscle occurred, requiring relief. Thus, complications occurred with 2 of the 43 screws implanted. In three patients, we observed non significant cement leakage into small veins (two patients) or out of the ventral sacral bone (one patient) in postoperative radiological controls, without any therapeutic consequences. No patient had neurological deficits or nerve root compression pain. Additional complications occurred in six patients; urinary tract infections in three, gastrointestinal infection with clostridia in one, pulmonary embolism in one. One patient with a Leriche syndrome not related to pelvic fixation procedure died after bypass surgery related infections 17 days after pelvic ring fracture fixation.

\section{Follow-up}

Twenty-eight patients were available for the 1-year follow-up. Five patients were unable to completely fill out the SF-12 because of impaired mental ability. One patient refused to participate in the follow-up. Two patients were lost to follow-up. Including the patient who died during hospitalisation, four patients died within 12 months after surgery. Hence, the data of 23/34 patients were available for analyses involving the followup examination.

\section{Quality of life (SF 12) and mobility}

The scores for the SF-12, neither for physical and mental state, did not significantly differ from that of the corresponding age- and gender-matched population of Germany $(p>0.05)$ [20]. The average physical composite score was $35.7 \pm 9.1$ points, and the average mental composite score was $48.3 \pm 11.8$ points. These results are graphically shown in Fig. 4 [20]. Nine patients were able to walk without orthopaedic aids after 1 year, six required one or two forearm crutches and 12 were using a walker. No patient was unable to walk.

\section{Pain}

The severity of pain at the time of admission was $6.7 \pm 1.4$ according to the VAS. One day before discharge, the severity of pain reduced to $2.7 \pm 1.0(p<0.001)$. The severity of pain was $3.4 \pm 2.3$ at the 1-year follow-up, which was not significantly different from that on the day of discharge $(p>0.2)$ (Fig. 5).

\section{Radiological follow-up}

$\mathrm{X}$-ray examination found no failures of SI screw fixation in any patient after 1 year.

\section{Discussion}

This prospective observational study investigated the clinical safety and 1-year follow-up results of percutaneous implantation of fenestrated SI screws with in-screw PMMA augmentation. The results show that this procedure was safe and had good clinical results, including
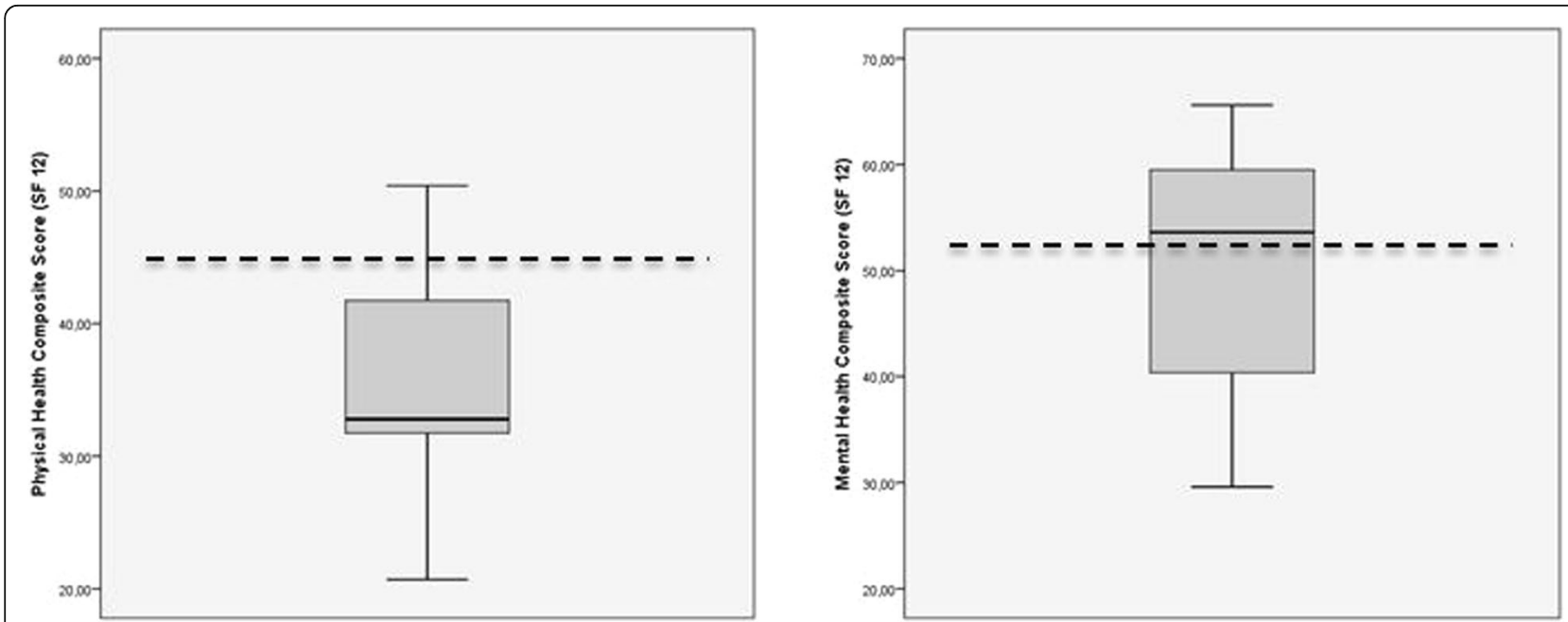

Fig. 4 Quality of life 1 year after in-screw cement-augmented SI screw fixation according to SF-12. Dotted line shows the average for an age- and gender-matched normal German population [20]. No significantly difference was found for the physical and mental score $(p>0.05)$ 


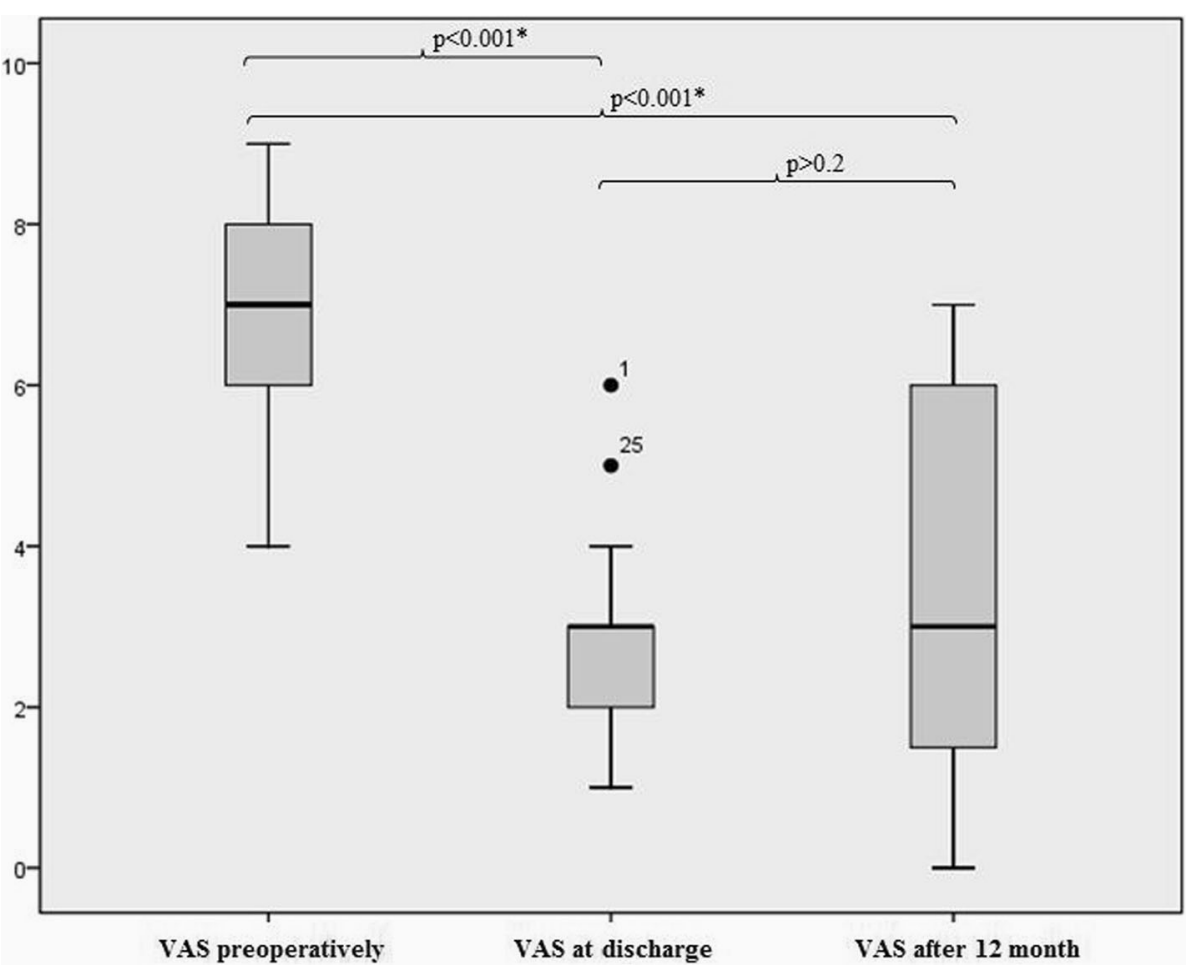

Fig. 5 Preoperative, postoperative and 1-year pain levels according to the visual analogue pain scale

significant pain reduction, as previously described in a short-term follow-up study in elderly patients $[14,17]$.

Because of the increasing numbers of fragility fractures of the pelvis, several surgical procedures have been introduced to ensure early ambulation $[2-4,6,7]$. To minimise perioperative risks and comorbidities and reduce operative time in elderly patients, percutaneous techniques have been frequently used [6].

In recent years, several percutaneous techniques have been presented to treat pelvic fractures in elderly patients. For example, the use of sacroplasty led to significant pain reduction and early ambulation but had no biomechanical advantages, as shown by cyclic loadings of cadaver pelves [15, 21, 22]. Nevertheless, fracture healing appears to be impossible after PMMA is added in the fracture gap. In our opinion, the combination of osteosynthesis and a SI screw and PMMA in-screw augmentation might provide more stability and still leave the fracture zone untouched for healing.

Other approaches include the use of a trans-sacral bar, an augmented transiliacal internal fixator or a combination of sacroplasty and SI screw fixation. All techniques have resulted in significant postoperative pain reduction and early mobility; however, they have only been investigated in studies with small sample sizes [16, 23, 24]. This study has also demonstrated a significant postoperative reduction of pain, thereby supporting the results of previous studies regarding in-screw cement augmentation [14, 17].
The short surgical time of $27 \mathrm{~min}$ per augmented screw demonstrates that the technique is minimally invasive [25]. Moreover, the rate of complications related to the augmented screws was not higher than that for non-augmented SI screws $[25,26]$. None of the complications described here were directly related to the use of PMMA and did not influence the outcome after discharge. Nonetheless, we did not use augmented screws for transforaminal sacral fractures because of a potentially higher risk for leakage of cement into the neuroforamina.

According to previously published reports, we also observed a long hospital stay of approximately 14 days and a high rate of adverse events during the hospital stay $[6,27]$. The reported 1-year mortality rate after sacral fragility fractures is up to $27 \%$, which is higher than our rate (4 of 34 patients) [28].

In our previous study we determined a significantly shorter hospital stay in the non-operative group compared to the operative group [29]. Nevertheless, approximately $20 \%$ of the non- operative group received surgery due to persistent pain and immobilization. This group most plausible corresponds to the patient population in the here presented study. In the two-year follow up there were no significant differences between all groups in pain level determined using the VAS. Moreover, as observed in the present study the physical state of all patients was lower compared to the age- and gender- 
adjusted German population but without achieving the level of significance. Compared to conservative treated patients also the hospital stay was shorter following conservative treatment but even shorter compared to other studies [29, 30]. Furthermore, also the complications might not differ between the different treatments and differences in deaths might be resulted from different sample sizes [29, 30].

Although these data might imply a missing superiority of the surgical treatment and especially the need for PMMA augmentation the observed higher survival following surgery might indicate a benefit by the surgical treatment [29]. Indeed, the parameters leading to this difference might be unclear and have to be elucidated. Even still alive the patient's life might be impaired so that permanent care might be necessary as reported for conservative treatment following a pelvic ring fracture in the elderly [6]. Data for the surgical groups are still missed and should be examined in future studies.

In consideration of these data $[6,29,30]$ and the excess of mortality following a pelvic ring fracture in the elderly [31], surgical treatment might be favored especially for patients suffering from persistent pain and immobilization following a conservative treatment [12].

But so far, due to osteoporosis in this patient population the rate of implant failure or backing out of the screws might be increased up to $14 \%$ as previously reported [12, 32]. Although the clinical consequence of this backing out is still unknown cement augmentation might be a promising technique to prevent this phenomenon. Thus, sacroiliac cement augmentation was introduced. Indeed, biomechanical examination failed to reveal the superiority of this technique in the hemipelvis model [33-35] the investigation of sole screw-sacral model yielded higher stability using augmented screws [36]. But so far, clinical trials using this technique especially regarding the outcome and pain are still sparse. Therefore, the present study was carried out.

A key finding is the pain reduction after 1 year with achieved mobility, which in our opinion, suggests the healing of the fracture. Without any proof, we assume reasons for the increasing distribution of the pain level after 1 year compared to discharge are co-morbidities (e.g. arthrosis, degenerative changes of the spinal column) and reduced or stopped analgesic therapy. Furthermore, the quality of life in this study population was not significantly different compared with that in the age- and gender- matched German population [20]. Moreover, in the present study no implant failure or subsequent pelvic ring fracture was observed. Regarding mobility there was no patient unable to walk suggesting also a minor amount of patients requiring permanent care.

Nonetheless, this study has some limitations. Although the study design was prospective, the rate of loss to follow-up was high. Different methods of osteosynthesis of the anterior pelvic ring might have influenced the clinical course. Because of the need for protection from radiation, not all patients underwent postoperative CT. One substantial limitation is the missed control group to compare the presented results.

\section{Conclusion}

In-screw PMMA augmentation of fenestrated SI screws is a safe technique with a short surgical time leading to pain reduction. Follow- up data suggests non-significant differences to the age- and gender-adjusted German population and no implant failure occurred. Although the superiority of this technique compared to conservative treatment or other surgical opportunities is not examined the presented procedure might be suitable after failed conservative treatment of fragility fractures of the sacrum.

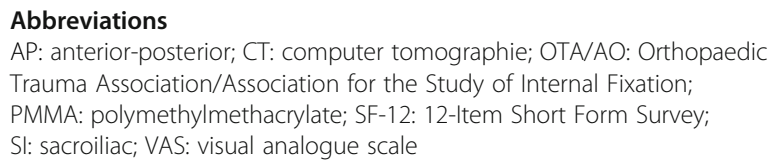

\section{Acknowledgements}

We acknowledge support from the German Research Foundation (DFG) and Leipzig University within the program of Open Access Publishing.

\section{Funding}

Within the program of Open Access Publishing the DFG and University of Leipzig pay the publication fee for this article. The founding sponsors had no role in the design of the study, in the collection, analyses, or interpretation of data or in the writing of the manuscript, and in the decision to publish the results.

\section{Availability of data and materials}

Raw and analysed data are available from the corresponding author on reasonable request.

\section{Authors' contributions}

AH, PP and JB drafted the protocol and edited the study design. JB lead the project group, JC and RH provided critical review of the manuscript and gave advisory for improvement. AH and PP wrote the manuscript. All authors read and approved the final manuscript.

\section{Ethical approval and consent to participate}

Ethical approval was obtained from the local ethics committee of the University of Leipzig (297-12-24,092,012) and, informed signed consent was obtained from all participating patients of the present study. Copies of the written consents are available for review by the editor of this journal.

\section{Consent for publication \\ Not applicable.}

\section{Competing interests}

Dr. Jörg Böhme has currently an advisory contract with Königsee Implants (Allendorf, Germany). All other authors declare that they have no conflict of interest.

\section{Publisher's Note}

Springer Nature remains neutral with regard to jurisdictional claims in published maps and institutional affiliations. 


\section{Author details}

'Department of Orthopedics, Trauma and Plastic Surgery, Spine Center, University Hospital of Leipzig, Liebigstraße 20, 04103 Leipzig, Germany. ${ }^{2}$ Department of Anatomy and Cell Biology, Martin Luther University Halle-Wittenberg, Grosse Steinstrasse 52, 06097 Halle (Saale), Germany. ${ }^{3}$ Present address: Clinic of Trauma, Orthopedic and Septic Surgery, Hospital St.Georg GmbH, Delitzscher Str. 141, 04129 Leipzig, Germany.

\section{Received: 7 June 2017 Accepted: 3 December 2017} Published online: 08 December 2017

\section{References}

1. Kannus P, Niemi S, Parkkari J, Palvanen M, Vuori I, Järvinen M. Nationwide decline in incidence of hip fracture. J Bone Miner Res. 2006;21:1836-8.

2. Buller LT. Best MJ, Quinnan SM. A Nationwide analysis of pelvic ring fractures: incidence and trends in treatment, length of stay, and mortality. Geriatr Orthop Surg Rehabil. 2016;7:9-17.

3. Breuil V, Roux CH, Carle GF. Pelvic fractures: epidemiology, consequences, and medical management. Curr Opin Rheumatol. 2016;28:442-7.

4. Andrich S, Haastert B, Neuhaus E, Neidert K, Arend W, Ohmann C, et al. Epidemiology of pelvic fractures in Germany: considerably high incidence rates among older people. PLoS One. 2015;10:e0139078.

5. O'Connor TJ, Cole PA. Pelvic insufficiency fractures. Geriatr Orthop Surg Rehabil. 2014:5:178-90.

6. Maier GS, Kolbow K, Lazovic D, Horas K, Roth KE, Seeger JB, Maus U. Risk factors for pelvic insufficiency fractures and outcome after conservative therapy. Arch Gerontol Geriatr. 2016;67:80-5.

7. Böhme J, Höch A, Boldt A, Josten C, Einfluss d. StandardComputertomografie hinsichtlich Frakturklassifikation und Therapie von Beckenringfrakturen bei Patienten über dem 65. Lebensjahr. Z Orthop Unfall. 2012;150:477-83.

8. Rommens PM, Hofmann A. Comprehensive classification of fragility fractures of the pelvic ring: recommendations for surgical treatment. Injury. 2013;44:1733-44.

9. Arduini M, Saturnino L, Piperno A, lundusi $R$, Tarantino U. Fragility fractures of the pelvis: treatment and preliminary results. Aging Clin Exp Res. 2015; 27(Suppl 1):S61-7.

10. Rommens PM, Ossendorf C, Pairon P, Dietz S-O, Wagner D, Hofmann A. Clinical pathways for fragility fractures of the pelvic ring: personal experience and review of the literature. J Orthop Sci. 2015;20:1-11.

11. Hegde V, Jo JE, Andreopoulou P, Lane JM. Effect of osteoporosis medications on fracture healing. Osteoporos Int. 2016;27:861-71.

12. Wagner D, Ossendorf C, Gruszka D, Hofmann A, Rommens PM. Fragility fractures of the sacrum: how to identify and when to treat surgically? Eur J Trauma Emerg Surg. 2015:41:349-62.

13. Tjardes T, Paffrath T, Baethis H, Shafizadeh $S$, Steinhausen E, Steinbuechel T, et al. Computer assisted percutaneous placement of augmented iliosacral screws: a reasonable alternative to sacroplasty. Spine. 2008;33:1497-500.

14. Wähnert D, Raschke MJ, Fuchs T. Cement augmentation of the navigated iliosacral screw in the treatment of insufficiency fractures of the sacrum: a new method using modified implants. Int Orthop. 2013;37:1147-50.

15. Garant M. Sacroplasty: a new treatment for sacral insufficiency fracture. J Vasc Interv Radiol. 2002;13:1265-7.

16. Mehling I, Hessmann MH, Rommens PM. Stabilization of fatigue fractures of the dorsal pelvis with a trans-sacral bar. Operative technique and outcome. Injury. 2012;43:446-51.

17. König MA, Hediger S, Schmitt JW, Jentzsch T, Sprengel K, Werner CML. In-screw cement augmentation for iliosacral screw fixation in posterior ring pathologies with insufficient bone stock. Eur J Trauma Emerg Surg. 2016;25:378.

18. Herman A, Keener E, Dubose C, Lowe JA. Simple mathematical model of sacroiliac screws safe-zone-easy to implement by pelvic inlet and outlet views. J Orthop Res. 2017;35:1478-84.

19. DVO Guidelines for the prevention, diagnosis and therapy of osteoporosis in postmenopausal women and men aged 60 and over. 2014. www.dvosteologie.org/dvo_leitlinien/osteoporose-leitlinie-2014. Accessed 13 January 2017

20. Ellert U, Bellach BM. The SF-36 in the Federal Health Survey-Description of a current normal sample. Gesundheitswesen. 1999;61:S184-90.

21. Kortman K, Ortiz O, Miller T, Brook A, Tutton S, Mathis J, Georgy B. Multicenter study to assess the efficacy and safety of sacroplasty in patients with osteoporotic sacral insufficiency fractures or pathologic sacral lesions. J Neurointerv Surg. 2013;5:461-6.
22. Mears SC, Sutter EG, Wall SJ, Rose DM, Belkoff SM. Biomechanical comparison of three methods of sacral fracture fixation in osteoporotic bone. Spine. 2010;35:E392-5.

23. Schmitz P, Baumann F, Grechenig S, Gaensslen A, Nerlich M, Müller MB. The cement-augmented transiliacal internal fixator (caTIFI): an innovative surgical technique for stabilization of fragility fractures of the pelvis. Injury. 2015; 46(Suppl 4):S114-20

24. Collinge CA, Crist BD. Combined Percutaneous lliosacral screw fixation with Sacroplasty using Resorbable calcium phosphate cement for osteoporotic pelvic fractures requiring surgery. J Orthop Trauma. 2016;30:e217-22.

25. Stöckle U, Schaser K, König B. Image guidance in pelvic and acetabular surgery-expectations, success and limitations. Injury. 2007;38:450-62.

26. Zwingmann J, Hauschild O, Bode G, Südkamp NP, Schmal H. Malposition and revision rates of different imaging modalities for percutaneous iliosacral screw fixation following pelvic fractures: a systematic review and metaanalysis. Arch Orthop Trauma Surg. 2013;133:1257-65.

27. Taillandier J, Langue F, Alemanni M, Taillandier-Heriche E. Mortality and functional outcomes of pelvic insufficiency fractures in older patients. Joint Bone Spine. 2003;70:287-9.

28. Morris RO, Sonibare A, Green DJ, Masud T. Closed pelvic fractures: characteristics and outcomes in older patients admitted to medical and geriatric wards. Postgrad Med J. 2000;76:646-50.

29. Höch A, Özkurtul O, Pieroh P, Josten C, Böhme J. Outcome and 2-year survival rate in elderly patients with lateral compression fractures of the pelvis. Geriatr Orthop Surg Rehabil. 2017;8:3-9.

30. Kanakaris NK, Greven T, West RM, van Vugt AB, Giannoudis PV Implementation of a standardized protocol to manage elderly patients with low energy pelvic fractures: can service improvement be expected? Int Orthop. 2017:41:1813.

31. Andrich S, Haastert B, Neuhaus E, Neidert K, Arend W, Ohmann C, et al. Excess mortality after pelvic fractures among older people. J Bone Miner Res. 2017;32:1789-801

32. Reuther G, Röhner U, Will T, Dehne I, Petereit U. CT-guided screw fixation of vertical sacral fractures in local anaesthesia using a standard CT. Rofo. 2014; 186:1134-9.

33. Osterhoff G, Dodd AE, Unno F, Wong A, Amiri S, Lefaivre KA, Guy P. Cement augmentation in sacroiliac screw fixation offers modest biomechanical advantages in a cadaver model. Clin Orthop Relat Res. 2016;474:2522-30.

34. Höch A, Schimpf R, Hammer N, Schleifenbaum S, Werner M, Josten C, Böhme J. Biomechanical analysis of stiffness and fracture displacement after using PMMA-augmented sacroiliac screw fixation for sacrum fractures. Biomed Tech (Berl). 2017;62:421-8.

35. Grüneweller N, Raschke MJ, Zderic I, Widmer D, Wähnert D, Gueorguiev B, et al. Biomechanical comparison of augmented versus non-augmented sacroiliac screws in a novel hemi-pelvis test model. J Orthop Res. 2017;35: 1485-93.

36. Oberkircher L, Masaeli A, Bliemel C, Debus F, Ruchholtz S, Krüger A. Primary stability of three different iliosacral screw fixation techniques in osteoporotic cadaver specimens-a biomechanical investigation. Spine J. 2016;16:226-32.

\section{Submit your next manuscript to BioMed Central and we will help you at every step:}

- We accept pre-submission inquiries

- Our selector tool helps you to find the most relevant journal

- We provide round the clock customer support

- Convenient online submission

- Thorough peer review

- Inclusion in PubMed and all major indexing services

- Maximum visibility for your research

Submit your manuscript at www.biomedcentral.com/submit
) Biomed Central 